University of Tennessee at Chattanooga

UTC Scholar

Honors Theses

Student Research, Creative Works, and

Publications

\title{
$5-2019$
}

\section{The effect of therapy dogs on preoperative anxiety}

Morgan Royer

University of Tennessee at Chattanooga, kgy625@mocs.utc.edu

Follow this and additional works at: https://scholar.utc.edu/honors-theses

Part of the Psychology Commons

\section{Recommended Citation}

Royer, Morgan, "The effect of therapy dogs on preoperative anxiety" (2019). Honors Theses.

This Theses is brought to you for free and open access by the Student Research, Creative Works, and Publications at UTC Scholar. It has been accepted for inclusion in Honors Theses by an authorized administrator of UTC Scholar. For more information, please contact scholar@utc.edu. 
The Effect of Therapy Dogs on Preoperative Anxiety

\author{
Morgan Royer
}

Departmental Honors Thesis Proposal

The University of Tennessee in Chattanooga

Psychology

Examination Date: November 12, 2018

Dr. Preston Foerder

Assistant Professor of Psychology

Thesis Director
Nicky Ozbek

Professor of Psychology

Department Examiner 


\begin{abstract}
Humans and animals have been companions for ages but that relationship and its benefits have only begun to be explored. Recently, animal assisted therapies have been used to improve both psychological and physiological wellbeing. One of the most popular therapies uses dogs. There are many research studies on the effects of therapy dogs on children and adults with results that have found that the companionship of a dog significantly decreases their stress levels in many different situations, including health care facilities. I investigated the effects of therapy dogs on adults' anxiety before going into day surgery. Participants were patients from the Erlanger Baroness Surgical Ambulatory Care Unit (SACU). Four conditions were compared to examine the effects of dogs on the patients' stress: therapy dog and handler team, stuffed dog and handler, a person only, or no person or dog. Before and after these visits the heart rate and blood pressure of the patient was taken. Patients also completed the State-Trait Anxiety Inventory short form (STAI) and a brief demographics survey. I found no change in blood pressure or heart rate in any of the conditions. I did find a statistically significant difference between the no person/no dog condition and any other intervention for the STAI scores, indicating that any intervention reduced anxiety. I also found that the stuffed dog significantly reduced anxiety as compared to no visitor. These results have implications for the effects of dog therapy on medical patients and provide avenues for future research.
\end{abstract}




\section{The Effect of Therapy Dogs on Preoperative Anxiety}

Humans and animals have been companions for ages and the presence of this bond in western culture is vast. The positive relationship between dogs and humans can be traced back over 10,000 years (Margolies, 1999). Many Western children's first toys and decorations are in the form of animals. These pictures become symbols of comfort for children throughout childhood (Serpell, 1999). Many children interact with animals in one way or another as they grow up. There are two types of animal interactions: long term and short term. A long-term interaction is owning and caring for a pet for many years. Short term interactions include animal assisted therapies (AAT). These therapies are short interactions with a dog for a specific reason to produce a certain benefit, such as reducing anxiety and increasing enjoyment of the situation. AAT is one of many forms of human-animal interactions (HAI) (Johnson, 2003).

Practitioners and physicians currently use HAI to reduce stress in many different settings such as counseling centers, schools, universities, doctors and dentists' offices, psychiatric facilities, hospitals, and many others. People of all ages and genders experience HAI treatments. HAI can be easily made to fit a variety of situations and is perceived positively by most individuals, making it an efficient method of reducing stress (Crossman, 2017). For example, it is common for colleges and universities to schedule a time for therapy dogs to come and visit with stressed college students before exams. These visits are not rigidly structured but rather offer a time for a student to interact with the dog. These interactions decrease stress in students that interacted with therapy dogs and that decreases stress associated with increased academic demands (Trammell, 2017). 
Anxiety is defined as a feeling of worry, nervousness, or unease, typically about an imminent event or something with an uncertain outcome. It is a form of stress and is characterized as a set of behaviors that can be organized under state and trait manifestations. State anxiety consists of the individual feeling tense, apprehensive, jittery, and worried, as well as physiological indicators of elevated heart rate and blood pressure for a short and specific period of time and certain situation. Trait anxiety is the experiencing of these symptoms over an extended period of time lending itself to becoming a trait of the person's personality (Caumo et al., 2001).

One form of state anxiety that is extremely common is "white coat syndrome" or experiencing anxiety due to being in a medical environment. During a medical examination, or other clinical situation, most individuals experience an increased level of distress. Simply being in the environment, hearing the sounds and seeing the equipment can induce anxiety in patients (Haugen et al., 2009). Experiencing surgery may also have longer term anxiety inducing qualities. In a peri-operative study, research that examines participants before, during and after the surgical experience, $28 \%$ of the participants experienced depression and/or anxiety symptoms prior to undergoing coronary artery bypass grafting (CABG), and $46 \%$ experienced symptoms after the CABG. These results show that, due to the surgery, patients experienced an onset of anxiety and depression. Major life changes, such as surgery, are state anxiety inducing factors that could eventually turn into a trait; hospitalization and surgery are examples of such changes (Li, Wang, \& Xiao, 2007).

One AAT that practitioners use to help their patients before and after surgery is therapy dog visits. The origin of therapy dogs is credited to Elaine Smith in 1976, a registered nurse. After seeing the effect a local chaplain's golden retriever had on her patients, she started the first 
official program for training therapy dogs, Therapy Dogs International (MacPete, 2018). Therapy dog visits are made possible by volunteer services and organizations; therefore, no additional cost or equipment is required of the staff or individuals receiving the therapy. The organization of this service itself is built to minimize stress on the facility and the individuals. (Marcus, et.al. 2013). These dogs are trained to be obedient, calm and comforting. Both handlers and dogs are required to pass specialized training, testing, and certification to insure the visits will be safe, positive, and non-disruptive to the facility. Dogs have the ability to identify the person most in need of therapeutic interaction and provide attention to that person. This ability may be explained by the dog's olfactory perception of chemical changes occurring as part of the human stress response (Marcus, 2013). A number of studies indicate that the presence of a dog can exert a positive influence on a child's psychological as well as physiological well-being (Johnson, Odendaal, \& Meadow, 2002; Moody, King, \& O’Rourke, 2002). In therapy dog visits to elementary classes, the presence of a calm dog companion can reduce stress levels in children. The physiological indicators of stress, such as elevated heart rate and blood pressure, can be reduced during and after interactions with the therapy dogs. (Katcher, Friedmann, Beck, \& Lynch, 1983).

The effect of the presence of an animal on human stress responses can be influenced by the familiarity of the setting, and the type of stressor. This means that an individual that is in a familiar setting and is experiencing a stressor to which they have previously been exposed will experience less stress than an individual in an alien environment who is experiencing a similar stressor. Another factor that could affect stress is the severity of the stressor and its potential to permanently affect the individual's life. Despite the variation that the stressors and settings may cause, when children were in a stressful situation such as reading aloud or a routine medical 
exam, the presence of a calm, attentive dog reduced the stress levels more than the presence of a strange adult, or even that of a friend (Friedmann, Thomas, \& Eddy. 2000). Therapy dog interventions have also been found to positively impact adults, as well. A group of adult fibromyalgia patients visiting an outpatient pain management clinic reported lower perceived pain, and distress, and improved mood after therapy dog visits (Marcus et al., 2013).

There are three different levels of HAI that provide physiological benefits to humans: a person looking at animals or pictures of animals; a person being in the presence of the animal but not physically interacting with it; and a person physically interacting with the animal (Friedmann et al., 2000). One study examined whether the presence of the dog itself was beneficial, or if even the smile of a friendly volunteer, without the presence of a dog would have the same benefit. The dog interaction with a quiet, non-interactive handler was found to be a more positive experience compared with quiet time and a friendly volunteer visit. (Johnson, Haubner, \& Sevedge, 2003).

I researched the effects of therapy dogs on adult anxiety in a preoperative setting. I used three control conditions, (a stuffed dog, a human, or time alone) to determine if a therapy dog condition was significantly more beneficial than the other levels of HAI. By creating different conditions, it was possible to narrow down which aspect of the visit was effective, whether it was the real dog's interaction and presence, the visual representation of a dog (as studied by the stuffed dog condition), having a person to distract and talk to them, or simply having 10-minutes alone with the person that brought them. I hypothesized that the interacting with a therapy dog would have a significant effect on lowering heart rate and blood pressure of the patients, as well as a significant positive effect on their state-anxiety level over the three control conditions. 


\section{Method}

\section{Participants}

Participants in this study were patients at the Erlanger Baroness Hospital Surgical Ambulatory Care Unit (SACU) in Chattanooga, TN. For a power of .80 and a medium effect size, we determined that we would require $N=104, n=26$ for each group. The actual number of patients in this study were 102 , as the volunteer condition only has 24 participants. Patients were selected at random from the patients that fit the criteria on the list of scheduled surgeries to be admitted in the SACU for the day of data collection. Patients were then randomly assigned to one of the four condition groups. Patients were not eligible for participation if they had canine allergies, were under the age of 18 or were above the age of 65 , were pregnant, a prisoner, cannot speak English, or did not wish to sign the informed consent form. All participants were accompanied by one family member or friend in the SACU room, as per usual protocol in the unit. All procedures with patients were approved by the UT College of Medicine IRB (Appendix B).

\section{Materials}

Spielberger State-trait Anxiety Inventory short form (STAI). This scale consists of a 6-item questionnaire. The state subscale asks questions to assess the how they feel "in the moment". Individuals rate their responses on a 4-point scale from 1 (not at all) to 4 (very much so). Scores range from 20 to 80 , with a higher score indicating a larger degree of anxiety. Testretest reliability for the state condition is less reliable than the trait condition, due to the nature of its acute condition (Bekker, 2015; Marteau \& Bekker, 1992; Speilberger et al., 1983;

Spielberger, et al., 1984) (see appendix A). 
Dinamap monitor. This device is a commonly used non-invasive oscillometric blood pressure and heart rate monitor. It is regularly used in the SACU to take routine vital signs. This device meets AAMI standards, however reliability is poor due to its tendency to overestimate the blood pressures. This device is best used under standardized conditions for the purpose of collecting change scores (GE Healthcare) (CARESCAPE VC150 - Patient Monitors - Patient Monitoring - Categories) (Beaubien, et al. 2002) (see Figure 1).

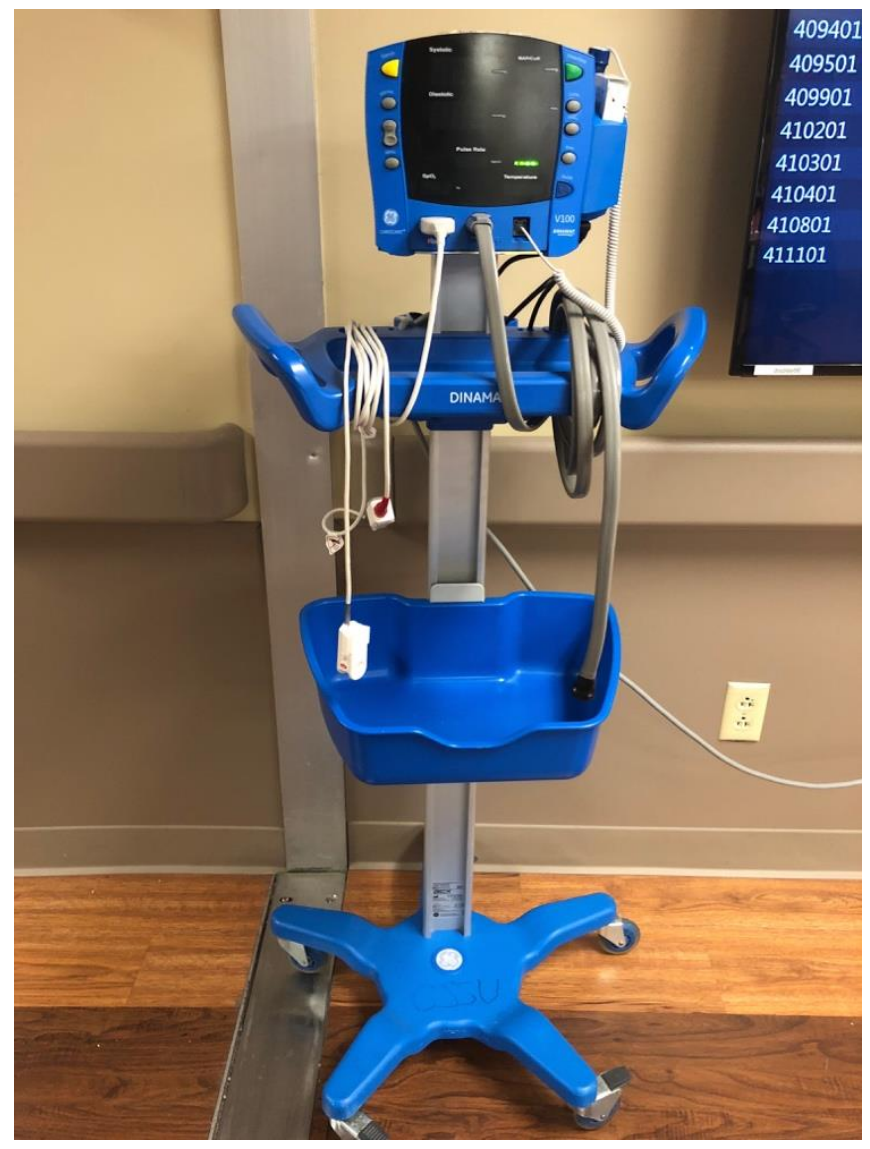

Figure 1. Dinamap Monitor

Stuffed dog. A Golden Retriever Giant Dog Stuffed Animal was used to represent the visual of a dog comparable in size and breed (Melissa \& Doug toy company), 31x35x12 in; 6lbs, (see Figure 2). This item was used during only the stuffed dog condition. 


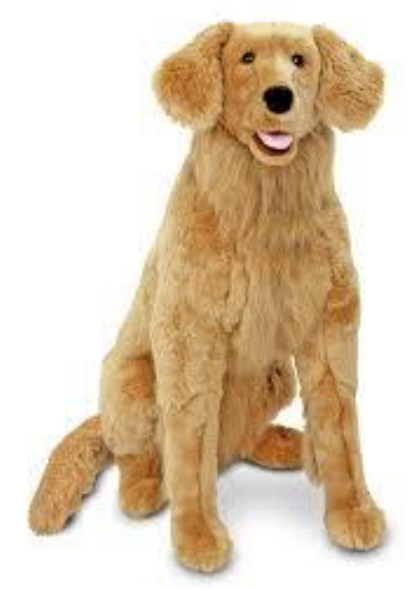

Figure 2. Stuffed Dog (Melissa \& Doug toy company)

Live Dogs. In this study, all dogs were golden retrievers, and were certified therapy dogs through Human Animal Bond in Tennessee (HABIT) who were already approved to visit the hospital and trained to be comfortable in the environment (see Figure 3). The three dogs were Monty, a 10-year-old male, Nugget, a 2-year-old female, and Reece, a 6-year-old male (see Figure 3). All procedures with dogs were approved by the University of Tennessee at Chattanooga (UTC) IACUC (Appendix C).
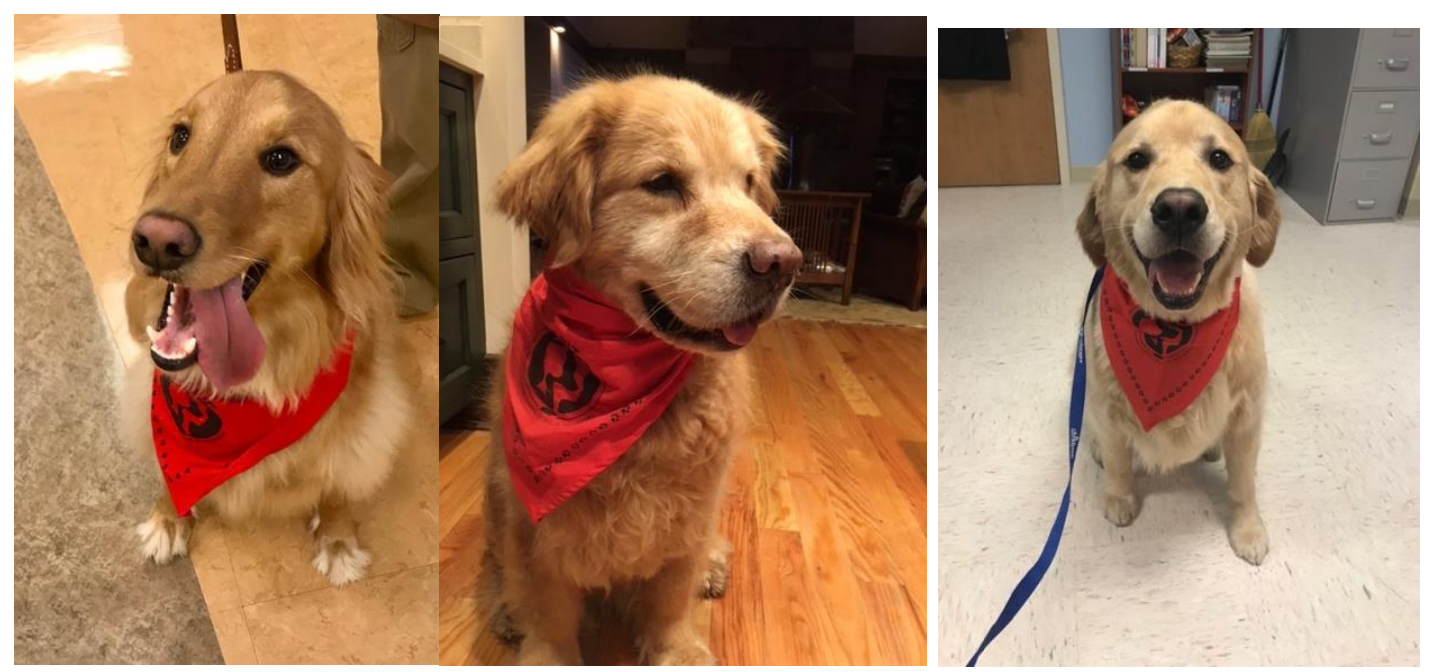

Figure 3. Nugget (left), Monty (middle), and Reece (right) 
Informed consent form. The informed consent form listed the procedures that the participants would experience, their rights and the study's procedures for anonymity. The consent form was reviewed and approved by the UT College of Medicine IRB (Appendix B).

\section{Procedure}

Patients were admitted to the SACU from the front desk and directed to a private room with the one individual that accompanied them. Upon admittance to the SACU all patients' vital statistics, including heart rate and blood pressure, were taken by a patient care technician, as per the hospital's usual preoperative procedure, including those used as the before ratings for heart rate and blood pressure. The randomly selected patients that met all criteria were presented with an informed consent form by a research assistant to indicate whether they chose to participate in the study. The study and consent form were explained in full, outlining the procedures, health information being taken, and the procedures in place to maintain anonymity. The patient was informed that if the research assistant or animal made them more uncomfortable, they could ask them to leave the room and their research participation would be terminated. However, this never occurred. Patients could decline participation or choose to participate. If they chose to participate, they then took part in the condition that they had been randomly assigned to.

The experiment used four conditions for 10-min sessions. Conditions were varied by day.:

Group 1. Control - no outside visitors enter the waiting room.

Group 2. Therapy Dog condition - a therapy dog team spends time in the room.

Group 3. Stuffed dog - a toy stuffed dog is in the room with a research assistant. Group 4. Volunteer - a research assistant with no dog is in the room with the patient. 
Once the patient agreed to participate and had signed the informed consent form, the researcher checked with the patient's assigned nurse to determine if it was an appropriate time for the 10-min session. If it was, the research assistant entered the room and began the session. The patient and their family member or friend stayed in their personal assigned SACU waiting room. At the end of the session, the participant's heart rate and blood pressure were taken for a second time. The participant was given the STAI (Appendix A) on paper while sitting in the SACU. The physiological measures were taken before STAI as psychological states change slower than physiological ones for the average person. After the patient completed the STAI, they were given a short demographics survey asking age, race, and gender. Once the survey was completed, and the patient was given a copy of the signed informed consent form, the research procedure was finished and the participants were taken to preoperative holding by Erlanger staff to continue with the regular course of treatment.

All research assistants were trained on data collection procedures by the lead investigator and all research procedures. The lead investigator was trained by a registered nurse on all procedures. All research assistants were female with varying physical appearance, college students with ages ranging from 20 to 22, and none of which received payment other than possible academic credit for the study.

Certified therapy dog handlers were likewise unpaid volunteers. Handlers were instructed in research procedure by the lead investigator. All handlers were women, varying in age and appearance. The PI was one of the dog handlers and also participated in collecting data in the other three conditions.

Group 1 experienced as close to a normal day in SACU as possible. Patients spent their 10 minute session in their SACU room with their family member or friend that they brought with 
them, as is usual procedure while waiting on surgery. They only changes made to their day were signing the consent form, experiencing the second measurement of blood pressure and heart rate, and filling out the STAI.

In the therapy dog condition (group 2) selected patients were visited by a therapy dog team, consisting of a trained volunteer and their certified therapy dog. The handlers were instructed to conduct the visit as they would any other, staying in the room and engaging the patient with the dog, while taking care not to speak of controversial or stressful subjects.

In the stuffed dog condition (group 3), a research assistant brought in a stuffed dog (see Figure 1) and stayed in the room for 10 minutes. The patient could choose to interact with the dog as they felt comfortable with. Research assistants were also instructed to avoid all controversial subjects, with the addition of redirecting the subject to the dog if conversation turned in a stressful direction.

For the volunteer condition (group 4), the research assistants were instructed to avoid high stress topics of conversation (politics, religion, or other controversial subjects), and be kind and polite to the patient and family member.

\section{Statistical analysis}

I analyzed the data using three contrasts and three separate linear regressions. Contrast 1 (C1) compared the no dog or person condition to all other conditions combined. Contrast 2 (C2) compared the volunteer condition to the combined stuffed dog and therapy dog conditions. Contrast 3 (C3) compared the stuffed dog to the therapy dog condition. Pair-wise post-hoc $t$-tests with a Bonferroni adjusted $\alpha=.017$ were used to compare group 1 to groups 2,3 , and 4 individually. 
Change scores were used to analyze the blood pressure and heart rate. Due to the nature of reporting blood pressure, mean arterial pressure (MAP) was calculated for each and then a change score was calculated from those. Mean arterial pressure is defined as the average blood pressure of the arterial part of circulation calculated by the systolic blood pressure (SBP) plus two times the diastolic blood pressure (DBP) all divided by three, $M A P=[S B P+2(D B P)] / 3$.

\section{Results}

We calculated the mean scores and standard error of the mean for all dependent variables and conditions (see Figure 4). We found a statistically significant score for STAI for C1. No statistically significant results were found for C2 or C3 for the STAI (see Table 1). In a post hoc comparison for STAI we found a statistically significant result for comparing group 1 and 3 (see Table 2). We found no statistically significant result for the physiological measures of heart rate or blood pressure (See Table 1 and 2).



Figure 4. Means for each measure for each condition. Error bars are SEM. 
Table 1

Results of Linear Regressions for All Comparisons

\begin{tabular}{llllllrr}
\hline & \multicolumn{2}{c}{ STAI } & \multicolumn{2}{c}{ BP Change } & \multicolumn{2}{c}{ HR Change } \\
\hline & $t$ & $p$ & $t$ & $p$ & $t$ & $p$ \\
\hline C1 & -2.403 & 0.018 & -0.501 & 0.617 & -0.145 & 0.885 \\
C2 & 0.822 & 0.413 & -1.275 & 0.206 & -1.066 & 0.289 \\
C3 & 0.747 & 0.457 & 1.124 & 0.264 & 0.590 & 0.557 \\
\hline
\end{tabular}

Table 2

Results of Post-hoc tests for All Dependent Variables

\begin{tabular}{|c|c|c|c|c|c|c|}
\hline & \multicolumn{2}{|c|}{$\underline{\text { STAI }}$} & \multicolumn{2}{|c|}{ BP Change } & \multicolumn{2}{|c|}{ HR Change } \\
\hline & $t$ & $p$ (1-tailed $)$ & $\mathrm{t}$ & $p$ (1-tailed $)$ & $t$ & $p$ (1-tailed) \\
\hline$(1,2)$ & 1.812 & 0.038 & -0.557 & 0.290 & -0.547 & 0.294 \\
\hline$(1,3)$ & 2.670 & 0.006 & 1.305 & 0.099 & 0.405 & 0.344 \\
\hline$(1,4)$ & 0.951 & 0.174 & 0.632 & 0.265 & 0.664 & 0.255 \\
\hline
\end{tabular}

\section{Discussion}

I hypothesized that the interacting with a therapy dog would have a significant effect on lowering heart rate and blood pressure of the patients, as well as a significant positive effect on their state-anxiety level over the three control conditions as indicated by the score on the STAI scale. The results did not support my hypothesis. I found no differences in heart rate or blood pressure. I found that participants that experienced anything or anyone visiting them (groups 2,3, and 4) reported statistically significant less anxiety than those who did not experience any extra visitation (group 1). The therapy dog condition did not have a statistically significant effect on 
the STAI score compared to nothing. These results were significant at $\alpha=.05$ but not once the post hoc adjustment was made. The therapy dog condition was not found to be significantly different than the others when compared to the other conditions, group 3 and 4 . The post-hoc comparisons for the STAI found that group 3, the stuffed dog group, reported statistically significant less anxiety than group 1 . These results do not agree with previous research done on reported anxiety, stress, and mood after therapy dog interactions (Marcuset al., 2013). Though group 3 is statistically significantly different from the control group, it was not found to be significantly different from the therapy dog condition (C3), indicating that there is no statistically significant difference between the effect of a live dog and a stuffed dog.

These results are different than those in previous studies that found that a therapy dog intervention was more beneficial than an interaction with a person or a stuffed dog (Johnson, 2003). Though the stuffed dog condition was found to significantly reduce anxiety, a visitor alone was not. This confirms that the presence of the form of a dog is what influenced the anxiety level of the patient, not the person in the room. I also found no statistically significant differences in the physiological measurements. This finding also contradicts other literature that found significant results (Katcher, Friedmann, Beck, \& Lynch, 1983). This could possibly be due to the high stress environment and situations that these patients were in compared to the other studies. Biological stress by be the result of physiological reaction to the fact that the body is about to be altered ( $\mathrm{Li}$, Wang, \& Xiao, 2007). Patients may have enjoyed the presence of the dog and perceived as beneficial, influencing their psychological well-being, thus the significant results for the STAI, but their physiology did not respond accordingly. Another point of consideration is that, normally, people are given a choice on whether or not they will see a therapy dog. In my study, they were randomly assigned to one of the four conditions. A patient 
who chooses to see a dog may have their mood lifted by the anticipation. The participants in this study may not have experienced that effect.

In terms of apparatus, more sensitive monitors may have been used to take data in previous studies as well. The Dinamap used is a common tool for preoperative check in procedures, and was chosen by convenience, but is not as exact as those used in actual surgery. A more sensitive device may detect a more significant change in the physiological stress indicators.

My results offer many options for hospitals to consider. All persons that entered the room were in regular, semiprofessional clothing, and were instructed to not talk about the surgery unless the patient mentioned it. And consistently across groups, it was found that anything entering the room was a significant improvement than no visit. This could be a sign that the uniforms of the nurses and doctors and other health care professionals induce "white-coat syndrome" (Fark, 1993). It may be that an intervention or policy changes within the perioperative systems, the processes that surround surgery (before, during and after), could reduce anxiety. The results show that even the representation of a dog can reduce anxiety of patients. It is possible that simply placing photos of dogs or stuffed animals could have a positive effect on patients as they wait for surgery.

There are many possibilities for future research. A few are: the effect of the breed of the dog, the length of time necessary for positive effects, like decreased anxiety and improved mood, to be seen, and research into other physiological measures as indicators of decreased stress. Different breeds have different reputations. Golden retrievers have a reputation of being sweet, calm and gentle (MacPete, Ruth. 2018). This study chose them out of convenience, as all therapy dog teams were volunteers, but they were a perfect choice for the study. The amount of time needed for a significant effect from therapy dog visit also needs to be studied. Therapy dog visits 
are usually very unorganized and the amount of time each person spends with the dog varies from person to person. There is much to learn about the effects of therapy dogs in all settings from empirical research. Humans have long lived their lives with the companionship of dogs and other animals (citation) and as we learn more about this companionship, more programs can be supported and created to utilize the relationship between animals and humans to enhance human life, whether it be in a medical environment or any other. 


\section{References}

Beaubien, E. R., Card, C. M., Card, S. E., Biem, H. J., \& Wilson, T. W. (2002). Accuracy of the Dinamap 1846 XT automated blood pressure monitor. Journal of Human Hypertension, 16(9), 647-652. doi:http://dx.doi.org/10.1038/sj.jhh.1001463

Bekker, H. L. (2015). Scoring 6 item STAI shortform. Retrieved from https://www.researchgate.net/publication/271700266_Scoring_6item_STAI_shortform_B ekker2015.

CARESCAPE VC150 - Patient Monitors. Retrieved from https://www.gehealthcare.co.uk/enGB/products/patient-monitoring/patient-monitors/carescape-vc150-vital-signs-monitor

Caumo, W., Schmidt, A. P., Schneider, C. N., Bergmann, J., Iwamoto, C. W., Bandeira, D., \& Ferreira, M. B. . (2001). Risk factors for preoperative anxiety in adults. Acta Anaesthesiologica Scandinavica, 45(3), 298-307.

Crossman, M. K. (2017). Effects of interactions with animals on human psychological distress. Journal of Clinical Psychology, 73(7), 761-784. doi:10.1002/jclp.22410

Davidson, A. T. (1984). Stress and surgery. Journal of the National Medical Association, 76(11), 1144-1145.

Fark, A. R. (1993). A pilot study of white-coat and labile hypertension: Associations with diagnoses of psychosocial dysfunction. Family Practice Research Journal, 13(1), 17-80.

Friedmann, E., Thomas, S. A., \& Eddy, T. J. (2000). Companion animals and human health: Physical and cardiovascular influences. In, Companion animals and use: Exploring the relationship between people and pets (pp.125-142). Cambridge: Cambridge University Press.

GE Healthcare. Retrieved from https://www.gehealthcare.co.uk/en-gb 
Haugen, A. S., Eide, G. E., Olsen, M. V., Haukeland, B., Remme, Å. R., \& Wahl, A. K. (2009). Anxiety in the operating theatre: A study of frequency and environmental impact in patients having local, plexus or regional anaesthesia. Journal of Clinical Nursing, 18(16), 2301-2310.

Johnson, R. A., Odendaal, J. S., \& Meadow, R. L. . (2002). Animal-assisted interventions research: Issues and answers. Westers Journal of Nursing Research, 24(4), 422-440.

Johnson, R. A., Meadows, R. L., Haubner, J. S., Sevedge, K. . (2003). Human-animal interaction: a complementary/alternative medical (CAM) intervention for cancer patients. Animal Behavioral Science, 47, 55-69.

Katcher, A. H., Friedmann, E., Beck, A. M. \& Lynch, J. J. (1983). Looking, talking and blood pressure: The physiological consequences of interaction with the living environment. In, New perspectives on our lives with companion animals, (pp. 351-359). West Lafayette, IN: Purdue University Press.

Li, X., Wang, Z., \& Xiao, F. (2007). Peri-operative depression and anxiety of coronary artery bypass grafting. Chinese Mental Health Journal, 21(3), 186-189.

MacPete, R. D. (2018). All About Therapy Dogs. [web log comment]. Retrieved from http://www.pethealthnetwork.com/news-blogs/a-vets-life/all-about-therapy-dogs

Marteau, T. M., \& Bekker, H. (1992). The development of a six-item short-form of the Spielberger State-Trait Anxiety Inventory (STAI). British Journal of Clinical Psychology, 31, 301-306.

Marcus, D. A. (2013). The science behind animal-assisted therapy. Current Pain Headache Reports, 17(4), 322. doi:10.1007/s11916-013-0322-2 
Marcus, D. A., Bernstein, C. D., Constantin, J. M., Kunkel, F. A., Breuer, P., \& Hanlon, R. B. (2013). Impact of Animal-Assisted Therapy for Outpatients with Fibromyalgia. Pain Medicine, 14(1), 43-51. doi:10.1111/j.1526-4637.2012.01522.x

Margolies, L. (1999). The long good-bye: Women, companion animals, and maternal loss. Clinical Social Work Journal, 27, 289-304.

Mellissa and Doug. (2018). Retrieved from http://www.melissaanddoug.com/golden-retrievergiant-dog-stuffed-animal/2109.html

Metzger, R. L. (1976). A reliability and validity study of the state-trait anxiety inventory. Journal of Clinical Psychology, 32(2), 276-278.

Miller, J., Connor, K., Deal, B., Duke, G. W., Stanley-Hermans, M., Varnell, G., Hartman, K., \& McLarty, J. . (2003). How animal assisted therapy affects discharge teaching: A pilot study. Critical Care Choices, 36-40.

Moody, W. J., King, R., \& O’Rourke, S. (2002). Attitudes of pediatric medical ward staff to a dog visitation program. Journal of Clinical Nursing, 11(4), 537-544.

Roberts, K. E. Factor structure and validity of the state-trait inventory for cognitive and somatic anxiety. Psychological Assessment, 28(2), 134.

Sandra B. Baker, J. S. K., Nancy L. McCain, Al M. Best. (2005). Measuring stress and immune response in healthcare professionals following interaction with a therapy dog: A pilot study. Psychological Reports (96), 16.

Serpell, J. (1999). Guest editor's introduction: Animals in children's lives. Society \& Animals, 7, $87-94$. 
Spielberger, C. D., Gorsuch, R., Lushene, R. Et., Vagg, PR., \& Jacobs, G. A.. (1983). Manual for the state-trait anxiety inventory (Form Y1 - Y2). Palo Alto, CA: Consulting Psychologists Press.

Spielberger, C. D., Gorsuch, R., Lushene, R., Vagg, P., \& Jacobs, G. (1984). State-trait anxiety inventory. Palo Alto, CA: Consulting Psychologists Press.

Trammell, J. P. (2017). The effect of therapy dogs on exam stress and memory. Anthrozoös, $30(4), 607-621$.

Zawistowski, S. (2008). Companion Animals in Society. Clifton Park, NY:

Thompson Delmar Learning. 


\author{
Appendix A \\ STAI State Short Form (Bekker, 2015). \\ Self-evaluation questionnaire (Y-6 item)
}

Name

Date

A number of statements which people have used to describe themselves are given below. Read each statement and then circle the most appropriate number to the night of the statement to indicate how you feel right now, at this moment. There are no right or wrong answers. Do not spend too much time on any one statement but give the answer which seems to describe your present feelings best.

\begin{tabular}{|c|c|c|c|c|}
\hline & Not at all & Somewhat & Moderately & Very much \\
\hline 1. I feel calm & 1 & 2 & 3 & 4 \\
\hline 2. I am tense .......... & 1 & 2 & 3 & 4 \\
\hline 3. I feel upset & 1 & 2 & 3 & 4 \\
\hline 4. I am relaxed & 1 & 2 & 3 & 4 \\
\hline 5. I feel content & 1 & 2 & 3 & 4 \\
\hline 6. I am worried & 1 & 2 & 3 & 4 \\
\hline
\end{tabular}


Appendix B

Informed Consent Form

\section{Consent for Participation}

University of Tennessee College of Medicine Chattanooga/Erlanger Health System

960 East Third Street

Chattanooga, Tennessee 37403

Title of Project: The Effect of Therapy Dogs on Preoperative Anxiety

Principal Investigator. Dr. Preston Foerder

Co-Investigator (5): Morgan Royer

Printed Name of Patient

\section{GENERAL INFORMATION:}

You are being asked to participate in the above titled climical research study conducted at Erlanger Health System. You have the option not to participate in this study. The study will last for approximately 12 months and there will be approximately 64 patients involved in this study.

\section{PURPOSE OF STUDY:}

The purpose of this research is as follows:

The purpose of this study is to examine the effects of interventions on the amxiety and stress of patients before surgery. This study will detemine whether therapy dogs significantly rechuce anxiety.

This research is being done because:

There is very little data on the physical and psychological effects of therapy dogs on human preoperative anxiety.

\section{DESCRIPTION OF STUDY:}

Each patient will be randomly selected to participate in the study. If you do choose to participate in the study you will stay in your SACU room with the individual you brought with you, as per usual protocol, for 10 minutes then asked to complete a brief survey. Your blood pressure and heart rate will also be taken before and after the condition is experienced.

- Procedures that are part of regular care and may be done even if not on the study. 


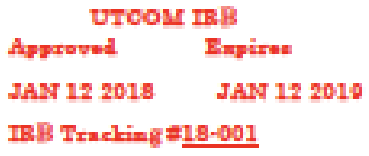

Blood pressure and heart rate will be taken as a regular preoperative procedure.

- Procedures being tested in this study.

Heart rate and blood pressure will be examined and compared in this study. The score on the survey will also be examined.

You may be joined during the ten minute period by a research assistant or by a live therapy dog or a facsimile of a dog. Please let us know if you would object to being in a room with any of these.

\section{RISKS OF RESEARCH STUDY:}

Risks and side effects related to the condition being studied include:

- Increase in anxiety

There also may be other side effects that cannot be predicted.

\section{BENEFTTS OF RESEARCH STUDY:}

There is no known benefit for participating in this study. However, information gained from this study may benefit patients in the future.

\section{CONFIDENTIALITY:}

Your research record will be labeled with a code number. A master key that links your name, personal information and the code number will be maintained in a separate and secure location. Individual subjects will not be identified in any presentations or publications based on the results of the research study.

\section{Your Privacy Rights}

Under federal privacy regulations, you have the right to decide who can see your personal health information (called "protected health information" or PHI). PHI collected in this study may include your medical history, your physical exams, lab tests, $x$-ray exams, and other diagnostic and treatment procedures, as well as information like your name, address, and date of birth. 


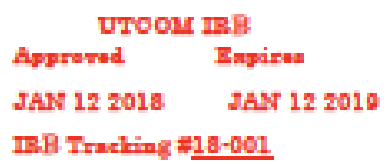

\section{Who Can See Your Records?}

By signing this consent form, you are letting the researchers at the University of Tennessee see your PHI collected in this study. In addition, other persons involved in doing and supervising this research may see your PHI, including researchers at University of Tennessee Psychology department.

\section{Who Protects Your Rights?}

The Institutional Review Board, (IRB), a group of people who review research work at the University of Tennessee College of Medicine Chattanooga IErlanger Medical Center, may review your PHI as part of its job to protect the rights and welfare of research subjects. Your PHI will not be shown to any other person: 1) except as required by law or, 2) for authorized oversight of this research study by other regulatory agencies or, 3 ) for other research which has been approved by the IRB. Your PHI will be used only for the research described in this consent form. Your PHI may be used until the study is completed.

\section{Can You Cancel this Consent?}

You may cancel this permission in writing at any time by contacting the principal investigator listed in the consent form PHI collected after your cancellation may not be used in the study. If you choose to cancel this permission, you may do so in person during the session or after by writing to the principal investigator at the following address:

\section{McCallie Ave, Dept. 2803, Chattanooga, TN 37403}

\section{What If You Refuse to Sign?}

If you refuse to provide this authorization, you will not be able to participate in the research study. If you cancel the authorization, then you will be withdrawn from the study. Finally, the federal regulations allow you to see your PHI collected or used in this study.

\section{COMPENSATION:}

There is no compensation for participating in this study. There is also no compensation for physical or psychological injury which you might incur as a result of this study. While medical care is available should an injury occur, the cost for such medical care will be your responsibility or your insurance company's responsibility and not the Investigator performing this study, the UT College of Medicine Chattanooga nor Erlanger Health System. 


\section{INVESTIGATOR COMPENSATION:}

The Principal Investigator (or Department Foundation) involved in this research will not receive compensation to cover administration costs associated with this research.

\section{CONTACT INFORMATION:}

If you have questions regarding your rights as a subject to this research, you should contact the UT College of Medicine Chattanooga/Erlanger Health System Institutional Review Committee at (423) $778-3818$

In the event of some medical problem, complication, injury or if you have questions regarding the research being performed, you should contact the investigator conducting the study: Morgan Royer (phone) 423-619-0916

This research protocol has been approved by the UT College of Medicine Institutional Review Board. 


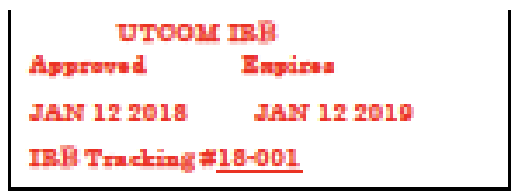

\section{PATIENT CONSENT}

I hereby freely and volumtarily consent to participate in the study described above. This consent is given based on the verbal and written information provided to me, and the understanding that I am medically and physically qualified to participate in this study. I am free to ask questions at any time.

I understand I have the option to choose not to participate or to withdraw from the study at any time without incurring any penalty or loss of benefits otherwise available to me, including medical care at this institution. I understand the investigator conducting this study may take me off the study if required in his/her discretion and judgement.

I understand there are inconveniences and possible complications which may occur related to participation in this study. These have been described both verbally and in this written consent form. I understand that by consenting to participate in this study, I am responsible for canying out instructions and that I must relate to the investigator, nurses, or study personnel any information that might be pertinent to the study, such as side effects of the treatment or procedure. I understand that any significant new findings or discoveries during the course of this research which may be related to my willingness to continue participation will be provided to me.

I understand that I will be given a signed and dated copy of this consent form.

Date/Time $\quad$ Printed Name of Patient

Date/Time Printed Name of Legal Rep
Representative

(Relationship)
Signature of Patient

Signature of Legal

I was present, heard, and observed the reading of this document and the explanation presented to the patient. I observed the signature of the patient or the patient's legally authorized representative and, in my opinion, I believe that the patient or representative understood the explanation presented and is voluntarily participating in the study without coercion.

Date/Time $\quad$ Printed Name of Witness
Signature of Witness 




I have carefully explained to the subject the nature of the above project. I hereby certify that to the best of my knowledge the person who is signing this consent form understands clearly the nature, demands, benefits, and risks involved in his/her participation and his/her signature is legally valid. A medical problem or language or educational barrier has not precluded this understanding.

Date/Time Printed Name of Staff Explaining Signature of Staff Explaining Consent

I certify that the Informed Consent was obtained correctly.

Date/Time Printed Name of Investigator Investigator

Signature of Principal 
Appendix C

IACUC Approval Letter

口MEUNIVERSITYof

TENNESSEE

CHATTANOOGA

Institutional Animal Care and Use Commintee

Dept 4915

615 Mecolie tivenue

Chathonogen TN 374n3-2586

Phone: (423) 425-5967

For: (423) 425,4060

iscucpoleds odu

MEMORANDUM

Hitplinumu utc eduiacuse

TO:

Dr. Preston Foerder

FROM:

Dr. Ethan Carver, LACUC Chair

Lindsay Pardue, Director of Research Integrity

DATE: January 25, 2018

SUBJECT: IACUC \#: 18-01: The Effects of Therapy Dogs on Preoperative Anxiety

The UTC Institutional Animal Care and Use Committee has reviewed and approved your application and assigned you the IACUC number listed above.

Reminder: Approved protocols must be reviewed at least annually. It is the responsibility of the principal investigator to submit an Application for Protocol Annual Continuation form to the IACUC before the anniversary date of the approved protocol. However, the Office of Research Integrity shall make every effort to send reminders 30 days prior to the anniversary date. The annual review form must be completed and submitted to the IACUC Committee before the first day of the anniversary month. New protocols must be submitted and approved every three years.

Please remember to submit a Protocol Modification Form if significant changes occur in your research design or in any instruments used in conducting the study. You should also contact the IACUC immediately if you encounter any adverse effects during your protocol.

For additional information, please consult our webpage http://www.utc.edu/lacuc or email lacucpro@utc.edu.

Best wishes for a successful research project. 